\title{
EFEITOS AGUDOS DO TREINAMENTO COM PESOS NA GLICEMIA: COMPARAÇÃO ENTRE TRÊS PROTOCOLOS PARA INICIANTES
}

\author{
Acute effects on blood glucose after weight training: \\ comparison of three protocols for beginners
}

\author{
Ricardo Yukio Asano1; João Bartolomeu Neto1; Marco Antonio Freitas Sousa'; Eduardo Fernandes de Miranda1 \\ ${ }^{1}$ Departamento de Educação Física do Centro Universitário UNIRG - Gurupi - TO, Brasil.
}

\begin{abstract}
Resumo: Os estudos relacionados aos efeitos do exercício no controle da glicemia, relatam adaptações positivas para saúde de sedentários e diabéticos, porém os efeitos agudos do exercício podem causar hipoglicemia principalmente em iniciantes.. Além disso, os estudos dos efeitos de exercícios específicos sobre a glicemia, como o treinamento com pesos são escassos. Portanto, o objetivo do presente estudo foi determinar os efeitos do treinamento com pesos na glicemia de sedentários, e comparar a alteração entre três protocolos para iniciantes em programas de treinamento com pesos. Metodologia: foram selecionados onze homens aparentemente saudáveis, com idade $24,5 \pm 8$ anos, peso $70,4 \pm 8,1 \mathrm{~kg}$, estatura 171,2 $\pm 5,1 \mathrm{~cm}$, índice de massa corpórea (IMC) $23,9 \pm 1,9$, porcentagem de gordura 15,3 $\pm 3,6 \%$. Os sujeitos realizaram três protocolos de treinamento com pesos: hipertrofia, resistência muscular localizada (RML) e força, com intervalo de duas semanas entre as sessões. Antes e após a realização dos protocolos foram coletas a glicemia, freqüência cardíaca e percepção subjetiva de esforço. Resultados: Em relação aos valores de repouso $(82,8 \pm 4 \mathrm{mg} / \mathrm{dL})$, a glicemia diminuiu significantemente $(p<0,05)$ em todos os protocolos. O protocolo de hipertrofia obteve diminuição significativa da glicemia $(64,3 \pm 2,5 \mathrm{mg} / \mathrm{dL})$, quando comparado com os protocolos de força $(74,6 \pm 5,2 \mathrm{mg} / \mathrm{dL})$ e RML $(70 \pm 3,7 \mathrm{mg} /$ $\mathrm{dL})$. Conclusão: $\mathrm{O}$ treinamento com pesos pode induzir indivíduos sedentários à hipoglicemia, principalmente o protocolo de hipertrofia utilizado neste estudo.
\end{abstract}

Palavras-chave: treinamento com pesos, glicemia, hipoglicemia.

Abstract: Studies of the effects of exercise on glycemic control, reported positive adaptations to health and sedentary diabetics, but the acute effects of exercise can cause hypoglycemia especially in beginners .. Furthermore, studies of the effects of specific exercises on blood glucose, and weight training are scarce. Therefore, the objective of this study was to determine the effects of strength training on glucose sedentary, and compare the change between the three protocols in programs for beginners weight training. Methodology: We selected eleven apparently healthy men, aged $24.5 \pm 8$ years, weight $70.4 \pm 8.1 \mathrm{~kg}$, height $171.2 \pm 5.1 \mathrm{~cm}$, body mass index (BMI) $23.9 \pm 1.9$, fat $15.3 \pm 3.6 \%$. The subjects performed three protocols of weight training hypertrophy, muscular endurance (RML) and strength, with an interval of two weeks between sessions. Before and after the implementation of the protocols were collected on blood glucose, heart rate and perceived exertion. Results: In relation to resting values $(82.8 \pm 4 \mathrm{mg} / \mathrm{dL})$, blood glucose decreased significantly $(\mathrm{p}<0.05)$ in all protocols. The protocol of hypertrophy achieved a significant decrease in blood glucose $(64.3 \pm 2.5 \mathrm{mg} / \mathrm{dL})$ compared with the protocols of force (74.6 $\pm 5.2 \mathrm{mg} / \mathrm{dL})$ and RML (70 $\pm 3.7 \mathrm{mg} / \mathrm{dL})$. Conclusion: Weight training can induce sedentary individuals to hypoglycemia, especially the hypertrophy protocol used in this study.

Keywords: training with weights, blood glucose, hypoglycemia.

Aceito em 27/10/2008 - Rev. Educ. Fís. 2010 - 03-09. Rio de Janeiro - RJ - Brasil

\section{INTRODUÇÃO}

Diminuição da glicemia é um evento comum durante o exercício com pesos ${ }^{(1)}$. Os músculos esqueléticos utilizam o glicogênio armazenado no processo de glicólise e metabolismo oxidativo para ressíntese do ATP. Posteriormente a diminuição da concentração de glicogênio durante o esforço físico implica na captação de glicose do sangue. O sistema neuroendócrino controla os níveis de glicemia em repouso e durante o esforço, buscando a prevenção da hipoglicemia principalmente a níveis de esforço acima do limiar anaeróbio, e ocorre por sincronização dos sistemas neural e endócrino que agem no fígado e no tecido adiposo(2,3). Contudo em exercícios com pesos moderados a intensos, a glicemia pode apresentar valores reduzidos, caso o volume de treinamento seja excessivo ${ }^{(1)}$.

Os sintomas de uma redução significativa da glicose sanguínea (hipoglicemia) induzem fraqueza, fome e vertigem, que afetam o desempenho nos exercícios e podem explicar parcialmente a fadiga 
do sistema nervoso central associado ao exercício com pesos ${ }^{(4)}$. Segundo Murray e Hackney ${ }^{(5)}$ a concentração de 70 mg.dL-1 de sangue ou valores abaixo, são considerados estado de hipoglicemia. Mas, o efeito hipoglicemiante do exercício físico, pode estimular adaptações crônicas positivas ao organismo. Estudos recentes comprovam os efeitos positivos do exercício na glicemia, principalmente em portadores de diabetes tipo I e tipo II e no aspecto da prevenção a essa doença ${ }^{(6,7,8,9)}$.

Algumas pesquisas foram direcionadas para determinar os efeitos do exercício com pesos nos mecanismo de controle da glicemia, na frequência cárdia e a percepção subjetiva de esforço. Os resultados encontraram adaptações crônicas positivas nos valores da glicemia no estado de repouso, na sensibilidade dos receptores musculares à insulina e na tolerância à glicose $\mathrm{e}^{(10,4,11)}$. Outra abordagem sobre exercício e glicemia a se considerar é a hipoglicemia induzida pelo exercício em iniciantes. Na revisão sobre hipoglicemia e exercício com pesos realizada por Brun ${ }^{(1)}$, foi observado que em indivíduos destreinados o risco de hipoglicemia no exercício é eminente. Hipoglicemia induzida pelo exercício é um exemplo de alterações fisiológicas do organismo que podem causar desconforto, e em caso de atletas diminuição da performance1. Porém, esta desordem pode acarretar adaptações ao organismo que podem ser benéficas, como o aumento da sensibilidade dos receptores de insulina na musculatura esquelética.

Estudos pesquisaram os efeitos de exercícios físicos específicos nos mecanismos de controle da glicemia. Como o treinamento com pesos, que parece diminuir a glicemia em repouso, aumentar estoques de glicogênio muscular, e apresentar efeitos agudos positivos para o portador de diabetes ${ }^{(12,2)}$.

Portanto, o objetivo do presente estudo foi comparar as alterações na glicemia, frequência cardíaca e na percepção subjetiva de esforço em sedentários aparentemente saudáveis e não diabéticos em três metodologias de treinamento com pesos.

\section{METODOLOGIA}

\section{População estudada}

Foram selecionados onze homens aparentemente saudáveis, com idade $24,5 \pm 8$ anos, peso $70,4 \pm 8,1 \mathrm{~kg}$, estatura $171,2 \pm 5,1 \mathrm{~cm}$, índice de massa corpórea (IMC) 23,9 $\pm 1,9$, porcentagem de gordura 15,3 $\pm 3,6 \%$ (TABELA 1). A seleção dos sujeitos incluiu indivíduos normotensos, nãodiabéticos, não-atletas e que não praticaram nenhum treinamento físico regular nos últimos doze meses. Os sujeitos selecionados formaram um único grupo experimental. Os indivíduos foram informados sobre o objetivo dos testes e assinaram termo de consentimento livre esclarecido, conforme determina a resolução 196/96 do Conselho Nacional de Saúde (CNS), sendo aprovado pelo comitê de ética em pesquisa da Fundação UnirG sob o protocolo no.0053/2009.

Tabela 1 - Medidas antropométricas da amostra

\begin{tabular}{lccccc}
\hline & $\begin{array}{c}\text { Peso } \\
\end{array}$ & $\begin{array}{c}\text { Estatura } \\
(\mathrm{kg})\end{array}$ & $\begin{array}{c}\mathrm{IMC}^{*} \\
(\mathrm{~cm})\end{array}$ & $\begin{array}{l}\text { \% Gordura } \\
\left(\mathrm{Kg} / \mathrm{m}^{2}\right)\end{array}$ & $\begin{array}{c}\text { Idade } \\
\text { (anos) }\end{array}$ \\
\hline Média & 70,4 & 171,1 & 23,9 & 15,3 & 24,5 \\
Desvio padrão & 8,1 & 5,1 & 1,9 & 3,6 & 8 \\
\hline
\end{tabular}

*IMC - Índice de massa corpórea

\section{Procedimentos metodológicos}

O grupo experimental realizou três protocolos diferentes de treinamento com pesos, com intervalo de duas semanas sem nenhum tipo de exercício físico entre os protocolos. O intuito do intervalo foi minimizar o efeito da adaptação ao exercício nos participantes da pesquisa ${ }^{(13)}$. Os protocolos foram baseados nas recomendações do American College of Sports Medicine para treinamentos com pesos para iniciantes e consistiam em: protocolo de força, protocolo de resistência muscular localizada (RML) e protocolo de hipertrofia respectivamente (TABELA $2)^{(14)}$. Os exercícios foram os mesmos nos três protocolos: Supino vertical, Fly máquina, Pulley, Voador, Hack horizontal, Flexão de perna, Extensão de perna, Panturrilha máquina, Máquina bíceps, Máquina tríceps.

$O$ teste de 1 repetição máxima (1 $\mathrm{RM}$ ) foi realizado para todos os exercícios, sendo que o procedimento se iniciou com um aquecimento geral de 4 a 5 minutos, seguido de um alongamento estático de baixa intensidade. $O$ aquecimento específico foi iniciado no aparelho, com carga aproximadamente de $50 \%$ de 1 RM prevista. O teste foi iniciado dois minutos após o aquecimento especifico. Foram orientados para executar duas repetições, caso fossem cumprisse as duas repetições na primeira tentativa, ou não completasse uma repetição. Uma 
segunda tentativa era iniciada após um intervalo de recuperação de três a cinco minutos com uma carga superior (primeira possibilidade) ou inferior (segunda possibilidade) àquela empregada anteriormente. Foi repetido uma terceira e ultima tentativa, caso ainda não se tivesse determinado a carga referente a uma única repetição máxima. No entanto, a carga anotada como 1RM foi aquela na qual foi possível ao sujeito executar somente uma única repetição. $O$ intervalo de transição entre os exercícios foi de três a cinco minutos.

Após o procedimento de coleta de $1 \mathrm{RM}$, foi realizado 8 repetições e em seguida a carga foi ajustada a $70 \%$ de 1 RM prevista e realizado 3 repetições. Os levantamentos subseqüentes foram realizados com apenas um movimento com cargas progressivas até a fadiga. A carga máxima foi considerada a maior carga com o movimento completo e realizado de forma correta. O intervalo de descanso entre as séries e as tentativas compreendeu entre 3 a 5 minutos $^{(15)}$.

A composição corporal foi determinada por meio do índice de massa corpórea (IMC), que foi calculada pelo peso corporal $(\mathrm{Kg})$ dividido pela estatura ao quadrado $\left(\mathrm{m}^{2}\right)(16)$. O peso corporal foi obtido em balança tipo plataforma, com capacidade de $180 \mathrm{~kg}$ $\mathrm{d}=50 \mathrm{~g}$, uma balança digital da marca G-TECH de uso pessoal, estando às pessoas descalças e com o mínimo de roupa possível. Para aferição da estatura utilizou-se o antropômetro livre da marca Sanny com precisão em centímetros, sendo mantida a pessoa em posição ortostática, com os pés juntos.

O percentual de gordura corporal (\%GC) foi obtido pelo somatório de 3 pregas cutâneas (peitoral, abdominal e coxa) de acordo com o proposto por Jackson e Pollock (1978 e 1980)(9). Considerou-se $\%$ GC elevado valores acima de $20 \%$ para os sexos masculino(10). As medidas das pregas foram obtidas utilizando-se o equipamento Lange Skinfold Caliper, sendo que cada uma foi verificada três vezes, ${ }^{(11)}$ trabalhando-se com a média dos dois valores mais próximos ou dos dois maiores quando os três valores eram consecutivos.

A alimentação das 12 horas que antecederam os testes foi padronizada por uma nutricionista para não alterar de forma sensível os valores habituais da glicemia em repouso. Duas horas antes do evento, os sujeitos realizaram uma refeição de 200 a 400 calorias dependendo da tolerância do indivíduo, com comidas habituais e ingestão de água.
Antes e imediatamente após os protocolos, foram determinados a glicemia, freqüência cardíaca e a percepção subjetiva de esforço.

\begin{tabular}{ccccc}
$\begin{array}{c}\text { Tabela 2 - Protocolo dos modelos de } \\
\text { treinamento de força utilizados no estudo }\end{array}$ \\
\hline Modelo & $\begin{array}{c}\text { Intensidade } \\
(\% \text { 1RM) }\end{array}$ & $\begin{array}{c}\text { Volume } \\
\text { (repetições) }\end{array}$ & $\begin{array}{c}\text { Tempo de } \\
\text { recuperação } \\
\text { (minutos) }\end{array}$ & $\begin{array}{r}\text { Velocidade } \\
\text { de contração }\end{array}$ \\
\hline Hipertrofia & $70 \%$ & 10 a 12 & $1: 30$ & Lenta \\
Força & $75 \%$ & 6 a 8 & $2: 30$ & Moderada \\
RML & $60 \%$ & 15 & $1: 00$ & Moderado \\
\hline
\end{tabular}

Fonte: American College of Sports Medicine14

A glicemia foi mensurada antes e após os protocolos. A coleta de sangue foi no dedo por perfuração e analisado pelo glicosímetro Prestigie System que possui validade de $\mathrm{r}=0,99$ com teste direto de glicemia Yellow Springs Instrument modelo $23^{\mathrm{a}(17)}$.

A freqüência cardíaca foi mensurada em repouso e após o término dos protocolos, através de freqüêncímetro da marca Polar.

A determinação da percepção subjetiva do esforço foi aplicado após o término dos protocolos. No momento da aplicação da escala (escala revisada de Borg), a tabela foi posicionada a frente do sujeito, e foi questionado qual o esforço percebido naquele momento ${ }^{(18)}$.

\section{Análise estatística}

Para a análise das variáveis dependentes (glicemia, percepção de esforço e freqüência cardíaca) e independentes (protocolos de força, hipertrofia e RML), utilizou-se do método estatístico ANOVA e teste LSD de Fisher ao nível de $p<0,05$. Primeiramente foi verificada a homogeneidade das variâncias através do teste Levene $(p=0,118)$. Utilizou-se $o$ aplicativo estatístico Bioestatistic 4.0.

\section{RESULTADOS}

A glicemia alterou significantemente ( $p$ $<0,05)$ nos protocolos de hipertrofia, força e RML, em relação aos valores de repouso. 
No treino de hipertrofia houve a maior diminuição $(64,3 \pm 2,5 \mathrm{mg} \cdot \mathrm{dL}-1)$ em relação aos níveis de repouso $(82,8 \pm 4 \mathrm{mg} / \mathrm{dL})$, chegando a níveis hipoglicêmicos. Protocolo de hipertrofia teve diminuição significativa ( $p$ $<0,05)$, quando comparado com protocolos de força $(74,6 \pm 5,2 \mathrm{mg} / \mathrm{dL})$ e RML $(70 \pm 3,7$ $\mathrm{mg} / \mathrm{dL}$ ). Já o protocolo de RML demonstrou diferença significativa $(p<0,05)$ quando comparado com protocolo de força, que menos alterou seus valores em relação aos níveis de repouso (FIGURA 1).

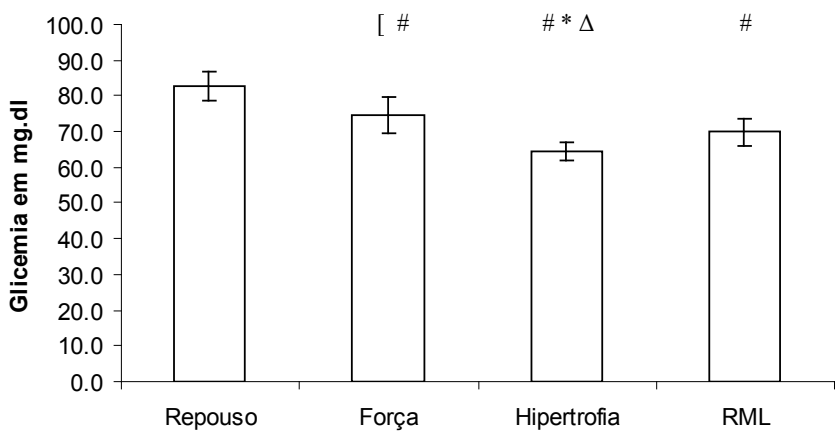

Figura 1 - Glicemia, em miligramas por decilitros (mg/dL), de sujeitos destreinados em treinamento com pesos, determinada em repouso e logo após o último exercício dos protocolos de RML, hipertrofia e força. Os resultados estão expressos como, média \pm desvio padrão, $(n=11)$, utilizando o nível de significância de $p<$ 0,05 , em comparação entre os protocolos.

\# - diferença significativa $(p<0,05)$, de repouso.

* - diferença significativa $(p<0,05)$, de força.

$\Delta$ - diferença significativa $(p<0,05)$, de RML.

[ - diferença significativa $(p<0,05)$, de RML.

Na freqüência cardíaca após treino, determinada imediatamente após o último exercício dos protocolos, verificou-se através do teste LSD que apenas não há diferença significativa ( $p$ $>0,05)$ entre as medidas de freqüência cardíaca, entre os protocolos de força $(98 \pm 5,3 \mathrm{bpm})$ e RML $(95 \pm 4,9 \mathrm{bpm})$. O protocolo de hipertrofia (121,2 \pm 7,4 bpm) obteve freqüência cardíaca significantemente maior quando comparado com RML e força (FIGURA 2). Protocolo de hipertrofia demonstrou aumento significativo da freqüência cardíaca $(p<0,05)$, quando comparado com repouso, protocolo de força e protocolo de RML.

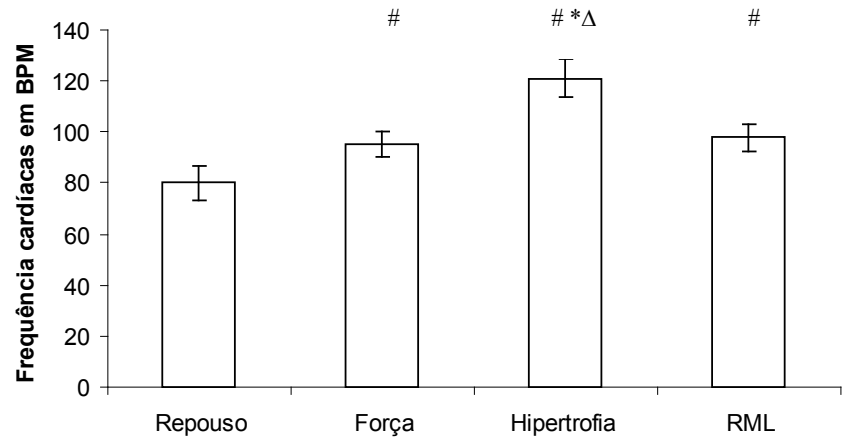

Figura 2- Freqüência cardíaca, em batimentos por minuto (bpm) de sujeitos destreinados em treinamento com pesos, determinada logo após o último exercício dos protocolos de RML, hipertrofia e força. Os resultados estão expressos como, média \pm desvio padrão, ( $n=11$ ), utilizando o nível de significância de $p<0,05$, em comparação entre os protocolos.

\# - diferença significativa $(p<0,05)$, de repouso.

* - diferença significativa ( $p<0,05$ de força.

$\Delta$ - diferença significativa $(p<0,05)$, de RML.

A percepção subjetiva de esforço (escala de Borg) foi medida em escala numérica de um a dez, demonstrou que os participantes do estudo relataram sensação de maior esforço nos modelos hipertrofia e RML em relação ao treino de força. Mensurada imediatamente após treino a média percepção de esforço foi de $4,9( \pm 0,9)$ para RML, 4,9 $( \pm 0,9)$ no treino de hipertrofia e 3,5 $( \pm 0,5)$ para força (FIGURA 3).

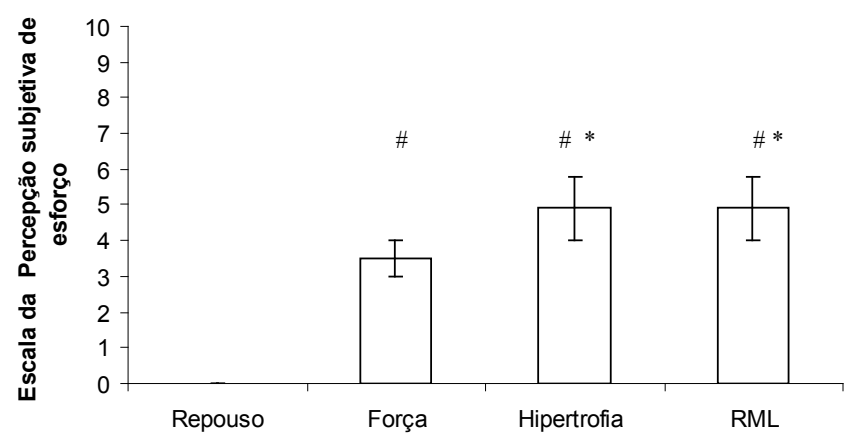

Figura 3 - Percepção subjetiva de esforço, de sujeitos destreinados em treinamento com pesos, determinada logo após o último exercício dos protocolos de RML, hipertrofia e força. Os resultados estão expressos como, média \pm desvio padrão, $(n=11)$, utilizando o nível de significância de $p<0,05$, em comparação entre os protocolos.

\# - diferença significativa $(p<0,05)$, de repouso.

* - diferença significativa $(p<0,05)$, de força.

\section{DISCUSSÃO}

Os protocolos de hipertrofia, RML e força, apresentaram diminuição da glicemia dos 
indivíduos em relação aos valores de repouso. O protocolo de hipertrofia comparado com os protocolos de força, RML e repouso apresentou diminuição significativa, alcançando valores considerados hipoglicêmicos.

Corroborandocomos resultados dopresente estudo, Brun et al(1) relata que em sedentários a produção de glicose hepática não é suficiente para manter níveis normais de glicemia pelo alto consumo de glicose pela musculatura esquelética, já que os principais substratos para exercício moderado para alta intensidade, são as vias da glicólise. A produção de glicose hepática em sedentários parece estar debilitada para situações como o exercício. Outros fatores de regulação da glicemia, também podem influenciar entre a produção e utilização de glicose pela musculatura esquelética, como por exemplo fatores hormonais, bioquímicos, transportadores de glicose na membrana muscular (GLUT 4) e duração do exercício.

Ferreira, Morel e Braga $^{(12)}$ também encontraram diminuição da glicemia após treinamento com pesos com intensidade de $65 \% 1 \mathrm{RM}$, que é correspondente ao protocolo de RML do nosso estudo, os indivíduos da pesquisa eram portadores de diabetes tipo I e a diminuição da glicemia após o treino foi de 22,2 $\pm 3 \%$ em relação a glicemia antes o treino.

Oliveira et $a^{(2)}$ realizaram um estudo com objetivo de analisar a possibilidade de determinar o limiar glicêmico e limiar de lactato utilizando exercícios resistidos. Os sujeitos da pesquisa realizaram exercícios com incrementos de carga progressiva, a glicemia diminui nas cargas iniciais até atingir o menor valor por volta de 30 a $36 \%$ de 1 RM (limiar glicêmico), e nas cargas posteriores a glicemia iniciou uma elevação. Os autores relacionaram o aumento da glicemia em cargas acima do limiar glicêmico com o aumento da glicogenólise e gliconeogênese induzida pelos hormônios adrenalina e glucagon, além da inibição de enzimas como a hexoquinase em função da diminuição do $\mathrm{Ph}$. Em contrapartida, nosso estudo utilizou as cargas relativas ao $1 \mathrm{RM}$ de $60 \%$ para RML, $70 \%$ para hipertrofia e $75 \%$ para força,. Comparando com os resultados do estudo citado, os protocolos estavam acima do limiar glicêmico (menores valores da glicemia) para treinamento com pesos, quando os mecanismos de controle da glicemia estão mais ativos, mesmo assim os treinos com pesos apresentaram diminuição da glicemia quando comparados com os valores de repouso. A explicação na discrepância nos resultados, pode estar no fato da pesquisa de Oliveira et al. ${ }^{(2)}$ ter utilizado um esforço progressivo até a exaustão e em nosso estudo o esforço foi intervalado com intensidade (carga) contínua.

Pascoe e Gladen $^{(19)}$, detectaram um aumento na taxa de ressíntese de glicose de 15.1 mMol.kg-1.min-1 para 33.6 mMol.kg.min, no treinamento com pesos. Esse tipo de exercício eleva substancialmente a utilização da glicose como fonte de energia. Com isso, a hipoglicemia pode ser um evento comum em iniciantes em programas de treinamento com pesos. Porém, as adaptações crônicas do treinamento com pesos podem ser positivas no controle da glicemia em repouso. Castaneda ${ }^{(20)}$ verificou que em indivíduos idosos após o período de treinamento com pesos realizado durante 16 semanas, houve diminuição da glicemia e aumento dos estoques de glicogênio muscular.

Os resultados do presente estudo apontam que os protocolos de RML e força, como esforços que menos alteram a glicemia de indivíduos destreinados em treinamento com pesos. Essa afirmação é importante em relação à prescrição do treinamento para iniciantes no sentido de minimizar o estresse induzido pelo exercício no início do programa de exercícios com pesos.

Já os efeitos do exercício físico em relação ao diabetes, as pesquisas demonstraram efeitos positivos no controle da glicemia ${ }^{(21)}$.

Willey e Singh (2003)(4) realizaram uma revisão bibliográfica sobre os efeito do exercício nos mecanismos de controle da glicemia e consideraram que o exercício aeróbio aumenta a sensibilidade à insulina e o controle da glicemia em pessoas idosas que possuem diabetes tipo II.

Kaln et al.(11) demonstraram aumento da sensibilidade à insulina, mas não ocorreu mudança no controle da glicemia durante 
treinamento aeróbio.

Sobre os efeitos do treinamento com pesos, Maiorana et al. ${ }^{(22)}$ demonstraram efeitos positivos no controle da glicemia em diabéticos tipo II, após treinamento em circuito com exercícios de força, que consistia em exercícios de baixa intensidade curto período de repouso e alto volume, juntamente com exercícios aeróbios.

Em relação aos efeitos agudos do treinamento, os efeitos hipoglicemiantes, também podem ser benéficos para o portador de diabetes tipo $\|^{(23)}$. Porém o estudo de Valle et al. ${ }^{(24)}$ alerta para os efeitos agudos do exercício para o diabético tipo II, em relação aos esforços realizados acima do limiar glicêmico, como o aumento abrupto da freqüência cardíaca e pressão arterial.

No presente estudo, todos os protocolos de treinamento com pesos testados diminuíram significantemente os valores da glicemia em relação ao estado de repouso. Portanto os dados sugerem que esses protocolos podem ser úteis em programa de exercícios físicos para portadores de diabetes. O protocolo de hipertrofia foi o que mais diminui os valores da glicemia, demonstrando que o consumo de glicose nesse modelo de exercício é elevado, principalmente quando comparado com os protocolos de força e RML.

Baseado nos estudos citados acima, podemos afirmar que o treinamento com pesos podem ser benéfico no mecanismo de captação de glicose pelos músculos esqueléticos, principalmente o protocolo de treinamento de hipertrofia, que neste trabalho consistiu em esforço de $70 \%$ de $1-\mathrm{RM}, 10$ a 12 repetições tempo de recuperação entre as séries de 1 minuto e velocidade de contração lenta. Porém os resultados da freqüência cardíaca e percepção subjetiva de esforço apontam o protocolo de hipertrofia como o maior esforço quando comparado com outros protocolos de força testados ${ }^{(25)}$. Essa informação é importante na prescrição do programa de treinamento com pesos para portadores de diabetes, uma vez que o perfil dessa população é em geral de baixa aptidão física e estilo de vida sedentária. Ou seja, os protocolos de treinamentos com pesos podem ser manipulados pelo profissional de Educação Física de forma a realizar uma progressão gradativa do esforço e aumentar a eficiência do programa.

Algumas variáveis foram fatores limitantes da pesquisa, como a impossibilidade de informações sobre os efeitos crônicos do treinamento com pesos na glicemia, pois a metodologia utilizada foi do método transversal e a não mensuração de mais variáveis dependentes, por exemplo alterações hormonais. Porém os resultados demonstraram a eficiência do músculo esquelético em difundir glicose do sangue para o interior de fibras musculares, que consiste no principal mecanismo debilitado do portador de diabetes, seja por insuficiência de insulina, ou a diminuição da sensibilidade à insulina dos receptores celulares. Portanto mais pesquisas podem ser realizadas no intuito de avançar esse conhecimento para otimizar e maximizar os programas de exercícios físicos para portadores de diabetes e minimizar os efeitos hipoglicemiantes em iniciantes em programas de treinamento com pesos.

\section{CONCLUSÃO}

A glicemia diminuiu significativamente nos três protocolos de treinamento em relação ao repouso, portanto a hipoglicemia em iniciantes de treinamento com pesos é eminente.

Dentre os protocolos estudados, o treinamento de hipertrofia foi o que mais induziu a hipoglicemia em sedentários, quando comparados com os protocolos de força e RML. Demonstrando que a prescrição e a periodização do treinamento com pesos devem levar em consideração as alterações metabólicas agudas, sendo assim o modelo de hipertrofia estudado deve ser inserido no programa de treinamento com pesos com maiores cuidados para iniciantes, ou em momentos mais avançados do planejamento.

Por fim, observou-se que em sedentários submetidos no treinamento com pesos, os valores diminuem significativamente ao final de uma sessão, porém o efeito hipoglicemiante dos treinamentos com pesos estudados podem ser benéficos para diabéticos. 


\section{REFERÊNCIAS BIBLIOGRÁFICAS}

1. Brun JF; Dumortier M; Fedou C e Mercier J. Exercise hypoglycemia in nondiabetic subjects. Diabetes Metabolic, 2001; 27(2): 92-106.

2. Oliveira JC, Baldissera V, Simões HG, Aguiar AP, Azevedo PHSM, Poian PAFO et al. Identificação do limiar de lactato e limiar glicêmico em exercícios resistidos. Rev Bras Med Esporte 2006; 12(6): 133-138.

3. Simões HG, Campbell CSG, Baldissera V, Denadai BS, Kokubun E. Determinação do limiar anaeróbio por meio de dosagens glicêmicas e lactacidêmicas em testes de pista para corredores. Rev paul educ fís. 1998; 12(1):17-30.

4. Willey KA. Singh MA. Battling insulin resistance in elderly obese people with type 2 diabetes: bring on the heavy weights. Diabetes Care, 2003; 26(5):1580-8.

5. Murray, RG.; Hackney, AC. Respostas endócrinas ao exercício e ao treinamento. In : Garrett JR, WE e Kirkendall, DT. A Ciência do exercício e dos esportes. Porto Alegre: Artmed, 2003.

6. Ross R, Dagnone D, Jones PJ, Smith H, Paddags $A$, Hudson $R$ et al. Reduction in obesity and related comorbid conditions after diet-induced weight loss or exercise-induced weight loss in men. A randomized, controlled trial. Ann Intern Med. 2000 ; 133(2):92-103.

7. Galassetti P, Tate D, Neill RA, Morrey S, Davis SN. Effect of Gender on Counterregulatory Responses to Euglycemic Exercise in Type 1 Diabetes. The Journal of Clinical Endocrinology \& Metabolism. 2002; 87(11):5144-50.

8. Briscoe VJ, Tate DB, Davis SN. Type 1 diabetes: exercise and hypoglycemia. Appl Physiol Nutr Metab. 2007; 32(3):576-82.

9. Fernandes Filho, J. A prática da avaliação física. $2^{\mathrm{a}}$ Ed.; Shape: Rio de Janeiro. 2003.

10. Mcardle WD, Katch FI, Katch VL. Fisiologia do exercício: energia, nutrição e desempenho humano. $5^{a}$ Ed.; Guanabara Koogan: Rio de Janeiro, 2003.

11. Kahn SE, Larson VG, Beard JC, Cain KC, Fellingram GW, Schartz RS. Effect of exercise on insulin action, glucose tolerance, and insulin secretion in aging. American Journal Physiology, 1990; 258: 937-43.

12. Ferreira BE, Morel EA, Braga PA. Alterações glicêmicas agudas em diabéticos tipo I após uma sessão de exercícios resistidos. EFdeportes, Buenos Aires 2008; 13(120):1.

13. Mujika I, Padilla S. Muscular characteristics of detraining in humans. Medicine Science in Sport and Exercise 2001 Aug; 33(8):1297-303.
14. Kraemer WJ, Kent A, Enzo C, Gary A D, Cathry D, Mattews $S \mathrm{~F}$ et al. Progression models in resistance training for healthy adults. Medicine and science in sports and exercise, 2002 Feb; 34(2): 364-380.

15. Brown, L.E., Weir, J.P. Recomendações de procedimentos da ASEP I: Avaliação precisa da força e potência muscular. Journal of Exercise Physiology. 4 (3): 01-21, 2001.

16. Costa, R.F. Composição corporal: teoria e prática da avaliação. São Paulo, Manole, 2001.

17. Hacket J, Aziz K. Review criteria assessment of portable blood glucose monitoring in vitro diagnostic devices using glucose oxidase, dehydrogenase methodology. FDA Draft Document, 1997; p-6

18. BORG, G. Escalas de Borg para dor e esforço percebido. Ed. Manole, 2000.

19. Pascoe DD, Gladden LB. Muscle glycogen resynthesis after short term, high intensity exercise and resistance exercise. Sports Medicine, 1996; 21(2): 98118.

20. Castaneda C, Layne JE, Munoz-Orians L, Gordon $\mathrm{PL}$, Walshimt J, Foldvari M et al. A randomized controlled trial of resistance exercise training to improve glycemic control in older adults with type 2 diabetes. Diabetes Care, December, 2000; 25(12): 2335-41.

21. Soukup JT, Kovalesk JE. A review of the effects of resistance training for individuals with diabetes mellitus. Diabetes, 1993; 19(4):307-12.

22. Maiorana A, O'Driscoll G, Goodman C, Taylor R, Green D. Combined aerobic and resistance exercise improves glycemic control and fitness in type 2 diabetes. Diabetes Clinic, 2002; 56(2) 115-23.

23. Kovisco VA, Yki-Jarvinem H, Defronzo RA. Physical training and insulin sensitivity. Diabetes Metab Ver. 1986; 1(4):445-81.

24. Valle G, Simões HG, Hiyane WC, Pardono E, Moreira SR, Ohata I et al. Respostas cardiovasculares de diabéticos tipo 2 durante exercício realizado em diferentes intensidades. Diabetes clinica. 2006; (4):, $271-6$

25. Asano, RY. Treinamento com pesos para iniciantes: comparação da incidência de microlesões em três modelos de treinamento. Revista de educação física. 2006:134: 22-9.

Endereço para correspondência:

e-mail-ricardokiu@ig.com.br, joaoefpira@hotmail.com, marcoafreitassouza@gmail.com, eduardounirg@gmail.com tel - (63) 8112-8108 ou (63)33122317- Coordenação de Educação Física UNIRG (63)3612-7612

End - Rua C-7, quadra 15 lote 02, Setor Canaã, Gurupi - TO, CEP 77400-000 